\title{
An experimental comparison of complex excitation sequences for eddy current testing
}

\author{
Giovanni Betta ${ }^{1}$, Pietro Burrascano ${ }^{2}$, Luigi Ferrigno ${ }^{1}$, Marco Laracca ${ }^{1}$, Marco Ricci ${ }^{2}$, Giuseppe Silipigni ${ }^{2}$ \\ ${ }^{1}$ University of Cassino and Southern Lazio, Dept of Eletrcical and Information Engineering, Via G. Di Biasio 43, 03043, Cassino (FR), Italy \\ ${ }^{2}$ University of Perugia, Dept. of Industrial Engineering, Loc. Pentima Bassa 4, 05100, Terni, Italy
}

\begin{abstract}
Eddy Current Testing (ECT) is a Non Destructive technique widely used in many industrial application fields in which it is very important to detect the presence of thin defects (generally called cracks) in conductive materials. Features of this technique are the costeffective implementation and the kind of retrieved measured data that make possible to estimate the geometrical characteristics of a crack as position, length, width and depth. The analysis of these characteristics allows the user to accept or discard realized components then improving the production chain. To accomplish for this task some aspects have to be taken into account during the measurement process. They mainly concern the realization of suitable measurement setup and post processing stages. As far as the measurement setup is concerned, crucial aspects are the choice of measurement and excitation devices. The choice of optimized excitation devices and strategies is of interest for research on Non Destructive ECT (ND-ECT): together with common aspects as the amplitude and the frequency of the exciting signal, the attention has been paid to issues as the type of signal to be adopted. In particular it has been found as the use of complex excitation signals, meant as signals different from the sinusoidal ones and with wide frequency content, might raise eddy current responses trying to support the measurement, detection and characterization stages when "difficult cases" are explored (i.e. very small and/or annealed cracks). In this paper the authors propose an experimental comparison of different excitation signals designed to improve the quality of experimental data when difficult cases are experienced and, consequently, to obtain a more reliable extraction of defects geometrical features.
\end{abstract}

Section: RESEARCH PAPER

Keywords: Extended Kalman Filter; fault diagnosis; on-line parameters estimation; Permanent Magnet Synchronous Generator; inter-turn short-circuit

Citation: Giovanni Betta, Pietro Burrascano, Luigi Ferrigno, Marco Laracca, Marco Ricci and Giuseppe Silipigni, An experimental comparison of complex excitation sequences for eddy current testing, Acta IMEKO, vol. 4, no. 1, article 19, February 2015, identifier: IMEKO-ACTA-04 (2015)-01-19

Editor: Paolo Carbone, University of Perugia

Received January $22^{\text {nd }}, 2014$; In final form December $5^{\text {th }}, 2014$; Published February 2015

Copyright: (C) 2014 IMEKO. This is an open-access article distributed under the terms of the Creative Commons Attribution 3.0 License, which permits unrestricted use, distribution, and reproduction in any medium, provided the original author and source are credited

Funding: The authors acknowledge financial support from Fondazione CARIT

Corresponding author: Giovanni Betta, e-mail: betta@unicas.it

\section{INTRODUCTION}

There are many techniques for the non-destructive inspection of conductive materials. Those based on the use of Eddy Current Testing (ECT) are probably the most widespread [1]. Key points of this kind of techniques are the relative low cost, the simple physical principle and hardware realization of the testing set-up.

Even if the physical principle that lies upstream of the ECT technique is very simple, its use for crack characterization purpose (i.e. evaluation of the geometric characteristics of the cracks) rather than for the only crack detection is a key issue in literature. In fact, many aspects have to be taken into account and suitably optimized during the overall measurement process. They mainly concern the realization of suitable measurement setup and post processing stages.

With respect to the measurement setup, the choice and the performance of measurement and excitation devices are of utmost importance. As for the former aspect, in the last years the literature have deeply explored physical principles and manufacturing techniques with the aim of realizing very good eddy current sensors and probes. Technologies as those based on coils, fluxgate, fluxset, Giant Magneto Resistance (GMR), hall effect and so on, have been analysed and adopted to realize ECT instruments. 


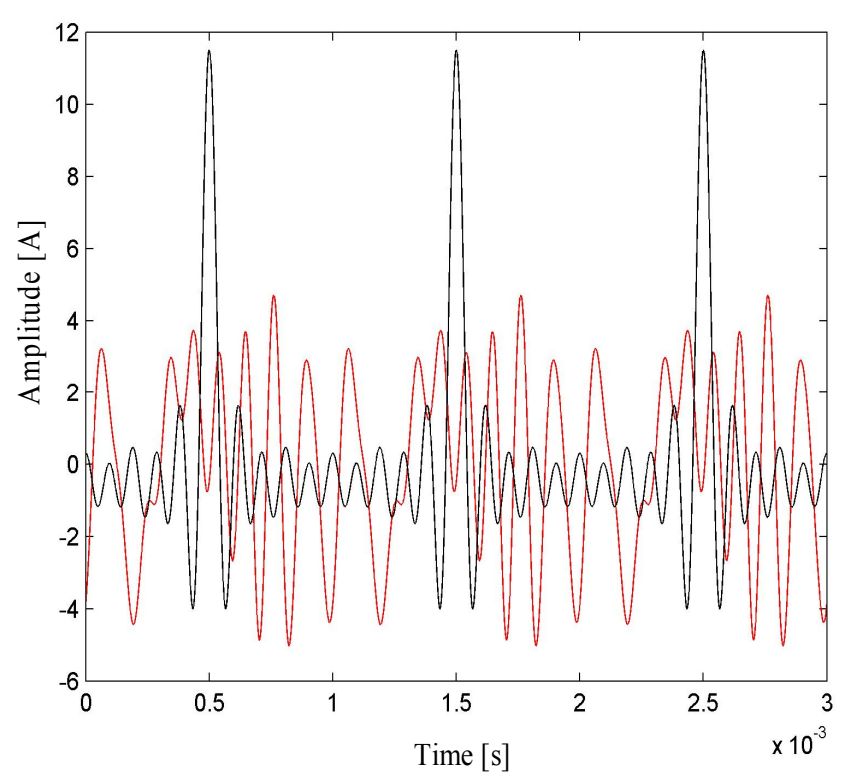

Figure 1. Example of a multisine signal composed by 10 sinusoidal tones with (red line) and without (black line) the phase optimization.

As far as the latter aspect is concerned, recent studies are involved in finding excitation solutions able to promote the measurement process. In this scenario, a key ingredient of the measurement process is the choice of signal type, frequency and amplitude to be adopted in the excitation stage. The criticality consists in finding excitation signals capable of causing sufficient amplitudes of eddy currents both for defects present on the surface of the material and for defects included in the material [2].

It is here reminded that the depth of eddy current is determined by the exciting frequency in such a way that the lower frequencies penetrate deeper than the higher ones, but, at the same stages, the lower the frequency the lower the amplitude of the reaction signal at the measurement probe. Suitable excitation signals are therefore required in order to achieve a compromise between the need of discover and characterize sub superficial defects and the need of obtaining good signal to noise ratio and repeatability of the measurement signals. In force of these considerations, the actual research on ECT is considering excitation signals containing wideband spectrums instead of the sinusoidal ones. To this aim some emerging techniques based on the use of multi-frequency, pulsed, chirp, and pseudo-noise signals, to cite a few, are proposed in literature as alternatives to traditional ECT signals in order to, primarily, improve the sensitivity of defect detection in some particular application field, and especially for small cracks, embedded deep in layered components [2-13]. Each one of these considered excitation signals present advantages and disadvantages that prevent the election of an absolute best excitation signal. The authors have engaged this field proposing probes, measurement methods and excitation strategies to optimize the defect characterization by ECT methods [2], [5], [6], [13], [14]. In this paper they present the extension of previous experimental studies [15] aimed in comparing two different excitation strategies
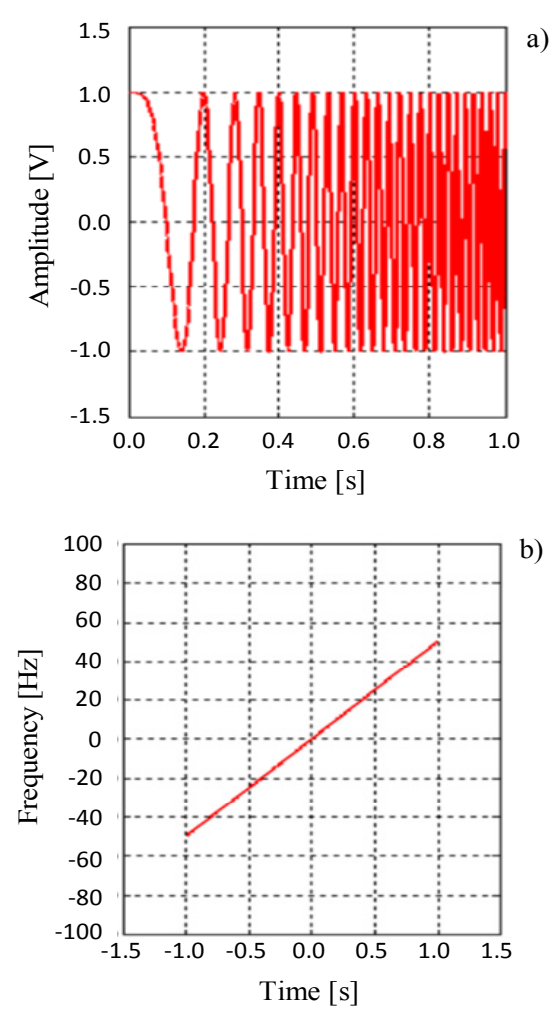

Figure 2. Time a) and frequency b) behavior of a linear chirp.

(multi-tone signal with optimized crest-factor and linear chirp) for the assessment of the above-mentioned characteristics of thin cracks in conductive materials. Although the two approaches are quite similar, there are some differences that make of interest to establish which technique provides better performances. In particular, while Chirp assures a higher information content than multi-tone, for both the continuous frequency range of excitation and the possibility to reconstruct also the impulse response, the multi-tone signal reduces significantly the complexity of data processing and also concentrates the energy in a discrete number of frequencies then assuring a high SNR for these values. At the same time the chirp signal, similarly to the Pulsed Eddy Current, allows the excitation of very low frequencies also for short signal duration.

In particular, the paper proposes an experimental approach based on the definition and analysis of a suitable figure of merit related to the detection capability.

Differently from previous works in the same topic [16] where different techniques were analyzed on the basis of the results of single measurements, here the comparison of the excitation performances is made by analyzing the images of the samples produced by various image formation procedures.

The final aim of the proposed study is toward the realization of a FPGA-based smart ECT measurement instrument able to automatically find the best excitation strategy depending on the defect characteristics. 


\section{THEORETICAL BACKGROUND}

Some ECT excitation strategies are experimentally analyzed and compared in this paper. They concern the use of multi-frequency and chirp signals.

\subsection{Multi-Frequency Signal}

A multi-frequency signal is realized combining some sinusoidal tones in order to excite the desired frequency components with the desired amplitudes. The general formulation of a multi-sine signal $I(t)$ is given hereafter (see equation 1).

$$
I(t)=\sum_{k=1}^{N_{S}} I_{k} \cdot \sin \left(2 \pi f_{k} t+\Phi_{k}\right)
$$

where $N s$ is the number of considered sinusoids, $I_{k}$ is the amplitude of the k-th sinusoid, $f_{k}$ and $\Phi_{k}$ are, respectively, the frequency and the phase of the $\mathrm{k}$-th sinusoid, $\pi$ is the pi Greek constant, and $t$ is the time.

With a proper choice of $\phi_{k}$,

$\phi_{k}=-\pi \frac{k(k-1)}{N_{S}}$

the resulting excitation exhibits a quite constant envelope, which is desirable for optimizing the power delivered to the exciting probe [17]. Figure 1 shows an example of a multifrequency signal composed by 10 tones up to $10 \mathrm{kHz}$ and with equal amplitudes, highlighting the effect of the phase optimization on the maximum values of the obtained signal. In addition, it is evident that the higher the number of tones the better the information retrieved from the eddy currents on the specimen. On the other hand, the higher the number of tones, the higher the total root mean square value of the generated signal. If this value is too high problems related to the excitation coil heating and the power supply dimensioning arise. For these reasons, generally, a finite number of tones is suitably chosen. Useful considerations concerning the relationships between the adopted frequencies and the specimen depth might drive the choice of tones number, frequencies and amplitudes.

\subsection{Chirp Signal}

Aforementioned, by tuning the amplitudes $I_{k}$ and the

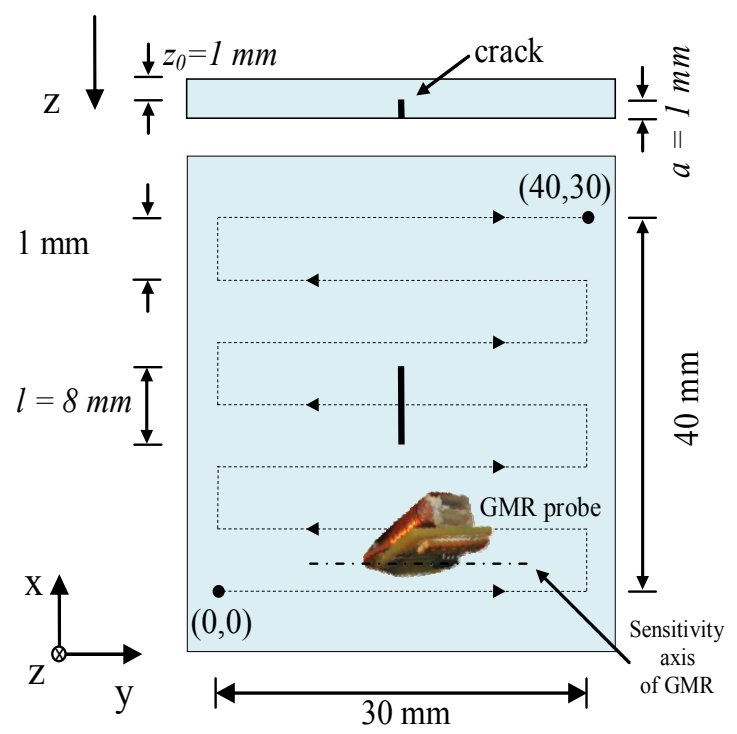

Figure 3. Example of the orthogonal scan for a specimen with sub superficial crack.

phases $\phi_{k}$, multi-tone signals can be generated in order to follow an arbitrary power spectrum while minimizing the peak factor. In the case of $N_{S}$ different harmonically related frequencies with constant power for each tone, the phases $\phi_{k}$ are derived from the continuous phase function of linear frequency modulated signals, i.e. from a Linear Chirp signal [17].

The Linear Chirp signal, also known as Sweep signal or Swept-frequency signals, is one of the most used waveform in pulse compression applications such as radar, sonar, spread-spectrum communications, etc. and it is described by the general expression:

$s(t)=\alpha(t) \cdot \sin (\Phi(t))$

where $\Phi(t)=2 \pi\left(f_{1} t+\frac{f_{2}-f_{1}}{2 T} t^{2}\right)$ is a quadratic phase term ensuring a linear sweep of the so called instantaneous frequency, $\quad f_{i}(t)=\frac{1}{2 \pi} \frac{d \Phi}{d t}=f_{1}+\frac{f_{2}-f_{1}}{T} t \quad$ and $\quad \alpha(t) \quad$ is a windowing function that is not-vanishing only in the interval $t \hat{I}[0, T]$ where $T$ is the signal duration. The concept of the instantaneous frequency is related to the spectrogram of the chirp signal and indeed on its power spectrum on short time intervals; $f_{1}$ and $f_{2}$ represent the initial and the

\begin{tabular}{|c|c|c|c|c|c|c|c|c|}
\hline \multicolumn{2}{|c|}{ 䓌 } & \multicolumn{2}{|c|}{ 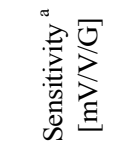 } & \multirow{2}{*}{ 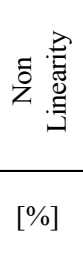 } & \multirow{2}{*}{ 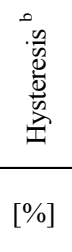 } & \multicolumn{2}{|c|}{ 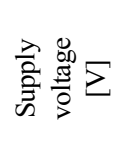 } & \multirow{2}{*}{ 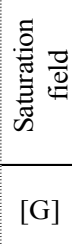 } \\
\hline 青 & 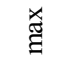 & 寻 & 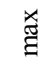 & & & 夆 & 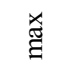 & \\
\hline 0.6 & 3.0 & 11 & 18 & 6 & $15 \%$ & \pm 1 & \pm 12 & \\
\hline
\end{tabular}

a. The sensitivity is defined as $\mathrm{mV} / \mathrm{G}$ for each volt of power supply b. For the unipolar operation.

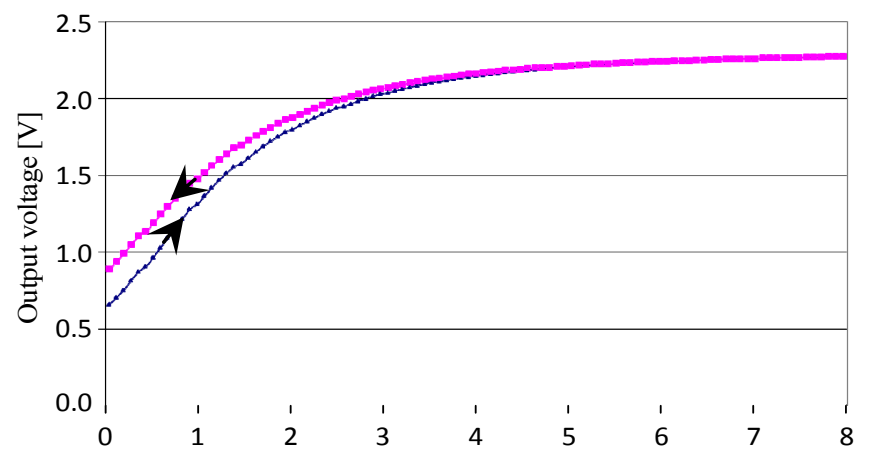

Applied DC magnetic field [G] 


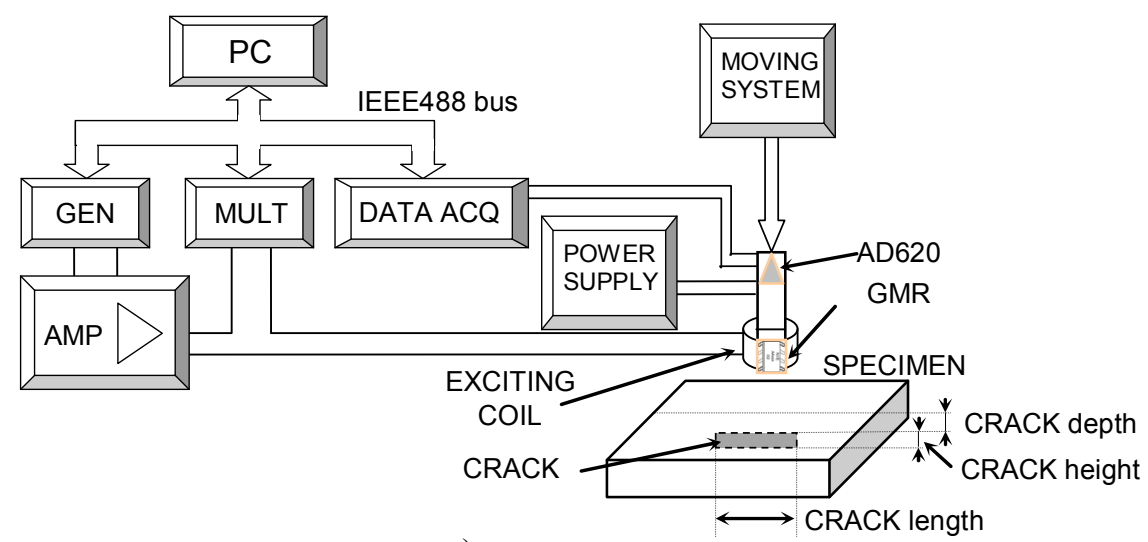

a)

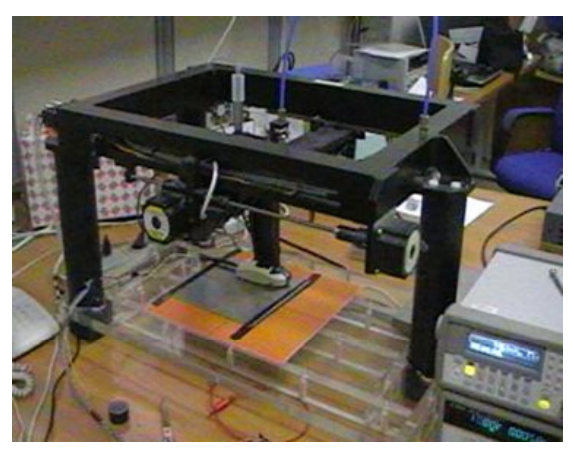

b)

Figure 5. The developed measurement station (a), and a photo that highlights the precision moving system (b).

final frequency values of the sweep. If $f_{1}<f_{2}$ the Chirp is named "up-chirp" otherwise, for $f_{1}>f_{2}$, it is called "downchirp". Inside the frequency range covered by the instantaneous frequency, i.e. $f \hat{[}\left[f_{1}, f_{2}\right]$ for an "up-chirp", the energy spectrum depends on the shape of $\alpha(t)$ that in the present case was chosen rectangular, i.e. $\alpha(t)=\theta(t)-\theta(t-T)$, in order to ensure a constant envelope and an almost flat power spectrum in the frequency interval.

More generally, $\alpha(t)$ can be used to modulate the amplitude of $s(t)$ and, due to the characteristic of the linear chirp, also the power spectrum. Figure 2a) shows the time behavior of a linear chirp while Figure $2 \mathrm{~b}$ ) shows the frequency variation over time.

By replacing the multi-tone signal with a chirp one, a continuous frequency range can be excited providing potentially a better resolution in evaluating the more sensitive inspection frequency for a given defect. Nevertheless, also the number of tones can be increased in the multi-tone signal to guarantee the wanted resolution so that the main advantage of the chirp excitation actually does not lie in its continuous frequency range of excitation but indeed is represented by the possibility to characterize contextually the time- and the frequency-domain response of the sample. In particular, besides of reconstructing the transfer function $H(\omega)$ in the frequency range of interest, the impulse response $h(t)$ of the sample can be retrieved by using a pulse-compression procedure. Since it was found that time- and frequency- analysis can provide complementary information about defects and moreover they are differently affected by the possible noise sources (environmental, lift-off variation, etc), the contextual analysis can improve the defect detection capability.

\section{THE MEASUREMENT SYSTEM}

In the following details about the adopted ECT probe together with a description of the measurement setup are given. The ECT probe is based on the use of a GMR sensor provided by NVE [18] and arranged in a Wheatstone bridge configuration [14], [19], [20].

Figure 3 shows a photo of the realized probe together with an example of the followed scan path for a subsuperficial crack with a depth of $1 \mathrm{~mm}$, a height of $1 \mathrm{~mm}$ and a length of $8 \mathrm{~mm}$. It is possible to highlight the presence of the GMR sensor in the lower part of the probe and the presence of the excitation coil in the upper part of the probe.

Figure 4a) shows the main features of the used GMR sensor while Figure $4 \mathrm{~b}$ ) shows its unipolar input/output characteristic.

The sensor has been arranged in the measurement setup sketched in Figure 5. It can be divided into generation and measurement sections. The generation section comprises the 20-20 Kepko ${ }^{\mathrm{TM}}$ Bipolar Operational Amplifier (AMP) driven by a frequency generator (GEN) and the exciting coil. The measurement section comprises: (i) the Agilent ${ }^{\mathrm{TM}}$ 34401A digital multimeter (MULT1) that measures both the AC and DC currents flowing in the exciting coil; (ii) the Analog Devices ${ }^{\mathrm{TM}}$ AD620 instrumentation amplifier, with a fixed gain equal to 10, whose output is measured by the Agilent ${ }^{\mathrm{TM}}$ 34401A digital multimeter (MULT2) that allows AC and DC voltage output of the sensor to be measured; (iii) a data acquisition system (DATA ACQ) based on the National Instruments ${ }^{\mathrm{TM}}$ NI USB 6212 allows the data digitalization and storage for the post-processing stage; (iv) a Keithley ${ }^{\mathrm{TM}} 2700$ digital multimeter (MULT3), fed by a K-type thermocouple, that monitors the GMR temperature. The whole measurement station is driven through a personal computer (PC) running an automation software written in the LabView ${ }^{\mathrm{TM}} 7.1$ environment. This software is able to generate the numerical multi-sine and chirp signals, to load the signals on the function generator and to acquire data from all the measurement devices present in the setup. Finally, a precision moving system moves the probe on the specimen following the selected path with a mean precision of $0.1 \mathrm{~mm}$. In particular, the data are collected on a regular grid of points of $31 \times 41 \mathrm{~mm}$ with a scanning step of $1 \mathrm{~mm}$.

All the experimental tests have been carried out on specimens with known defects. All the specimens are plates realized in 2024-T3 aluminum alloy. They are square plates characterized by a length $20 \mathrm{~cm}$ and thicknesses equal to 2 $\mathrm{mm}$. The thin defect is located at the center of each plate. 

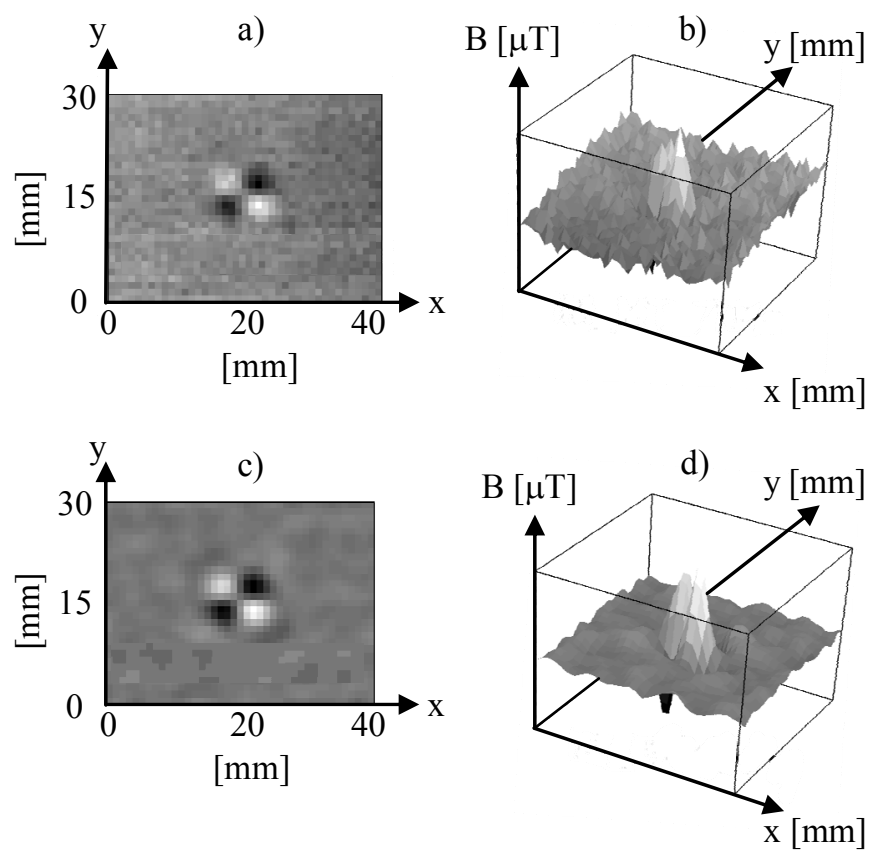

Figure 6. (a) Normalized image for the multi-frequency stimulus at the exciting frequency of $7 \mathrm{kHz}$; (b) map, in the three-dimensional space, of the image sketched in (a); (c) effect of the band pass filtering procedure on the image sketched in (a), and (d) map, in the three-dimensional space, of the filtered image.

Each defect has a width equal to $0.1 \mathrm{~mm}$. Details on each specimen are reported in Table I. A total of 5 specimens have been analyzed.

\section{RESULTS}

Different post-processing algorithms have been applied to both the multi-sine and chirp signals. In the following the adopted processing is described in detail.

\subsection{Post processing for the multi-frequency signals}

The voltage proportional to the reaction magnetic field acquired by the ECT probe has to be processed in order to extract the material response to the multi-frequency stimulus. A Goertzel based algorithm has been developed to estimate the field amplitude and phase values for each frequency component of the acquired signal. In this way an electromagnetic response of the material at different frequencies is obtained.

For each frequency $\left\{f_{\alpha}, \alpha \hat{I}[1,10]\right\}$ a $2 \mathrm{D}$ image that visualizes for each point $\{(x, y): x \hat{I}[1,41], y \hat{I}[1,31]\}$ the amplitude of the "normalized" transfer function $\left|\hat{H}\left(f_{\alpha}, x, y\right)\right|=\left|H\left(f_{\alpha}, x, y\right) / H\left(f_{\alpha}, 1,1\right)\right|$ is formed.

A normalization procedure is made with respect to the transfer function measured on a healthy point of the sample

Table 1. Geometrical characteristics of the adopted specimen; each crack has a width equal to $0.1 \mathrm{~mm}$.

\begin{tabular}{|c|c|c|c|c|c|}
\hline \multirow{2}{*}{$\begin{array}{l}\text { Crack } \\
\text { characteristics }\end{array}$} & \multicolumn{5}{|c|}{ Sample } \\
\cline { 2 - 6 } & $\# 1$ & $\# 2$ & $\# 3$ & $\# 4$ & $\# 5$ \\
\hline Length [mm] & 1.0 & 5.0 & 5.0 & 5.0 & 8.0 \\
\hline Height [mm] & 1.0 & 1.6 & 1.0 & 0.4 & 1.0 \\
\hline Depth [mm] & 1.0 & 0.4 & 1.0 & 1.6 & 1.0 \\
\hline
\end{tabular}
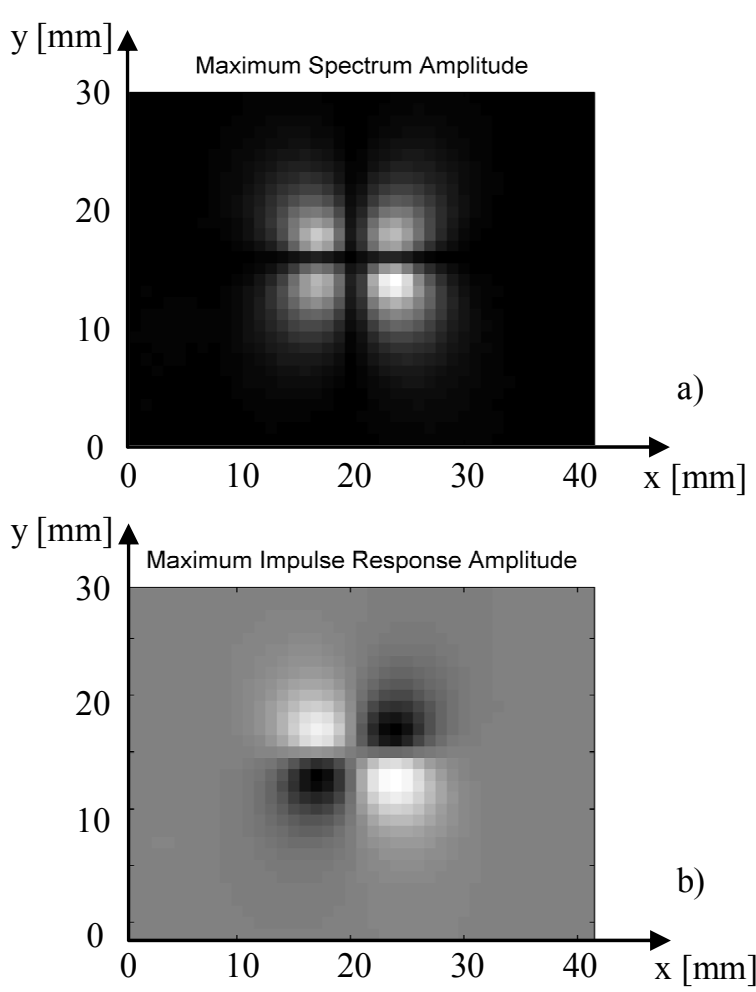

Figure 7. Normalized image for the chirp stimulus by applying the IM_CH_T (a) and IM_CH_F (b) processing for the sample \#4 after the application of the filtering procedure.

(in this case the first scanned point). For each sample $N s=10$ images are then obtained, henceforth labelled as IM_MTakHz. A 2D band pass filter acting as noise smoothing in the region of interest is applied to reduce the effect of the environmental noise, thermal drifts in components and lift-off variation. It is worth noting that for small inner defects, the noise can be comparable with the signal and worsening the SNR of the data and of the images.

For example considering the Sample \#4 and the exciting frequency of $7 \mathrm{kHz}$, figure 6 shows the effect of a filtering procedure. It is possible to highlight as the filter reduces the effect the low frequency variations of the image intensity as well as high frequency components corresponding to the effect of AWGN. It can be noted that the defect is more visible after the filtering process.

\subsection{Post processing for the chirp signals}

To extract the data of interest from the acquired chirp signal, different strategies can be implemented. The authors have explored three different strategies typically adopted in processing chirp signals that are described in the following.

i) For each point $(x, y)$, the modulus of the "normalized" transfer function $|\hat{H}(f, x, y)|=|H(f, x, y) / H(f, 1,1)|$ is calculated and subdivided in $N s=10$ adjacent frequency regions each of them centered at the tone $f_{\alpha}$ with $\alpha \in[1,10]$. Then, similarly to the multitone case, for each sample $N s=10$ images are obtained, henceforth 
labelled as IM_CH_akH. For all the considered images pixels correspond to the maximum of the normalized transfer function in the $\alpha-$ th bin:

$$
\begin{aligned}
& \text { IM_CH_akHz}(\mathrm{x}, \mathrm{y})=\ldots \\
& \ldots \max _{\mathrm{f}}\{|\hat{\mathrm{H}}(\mathrm{f}, \mathrm{x}, \mathrm{y})|, \mathrm{f} \in[\alpha \mathrm{kHz}-\Delta, \alpha \mathrm{kHz}+\Delta]\}
\end{aligned}
$$

In this case $2 \Delta=1 \mathrm{kHz}$ is the bin width.

ii) For each point $(x, y)$ the "normalized" impulse response $\hat{h}(t, x, y)=h(t, x, y)-h(t, 1,1)$ is calculated by applying pulse compression. The image IM_CH_T is then formed by taking for each point the maximum of the impulse response:

$$
\mathrm{IM}_{-} \mathrm{CH}_{-} \mathrm{T}(\mathrm{x}, \mathrm{y})=\max _{\mathrm{t}}\{\hat{\mathrm{h}}(\mathrm{t}, \mathrm{x}, \mathrm{y})\}
$$

iii) For each point $(x, y)$ the Chirp-Z-Transform $\hat{\mathrm{D}}(\mathrm{f}, \mathrm{x}, \mathrm{y})=\mathrm{CZT}[\hat{\mathrm{d}}(\mathrm{t}, \mathrm{x}, \mathrm{y})]$ of the "normalized" output signal is calculated between $f \in[0.5 \mathrm{kHz}, 10.5 \mathrm{kHz}]$ and then the image IM_CH_F is formed by taking for each point the maximum of the Chirp-Z-Transform:

$$
\left.\mathrm{IM}_{-} \mathrm{CH}_{-} \mathrm{F}(\mathrm{x}, \mathrm{y})=\max _{\mathrm{f}}\{\hat{\mathrm{D}}(\mathrm{f}, \mathrm{x}, \mathrm{y})]\right\}
$$

While IM CH T and IM $\mathrm{CH} F$ are characteristic for the chirp since they exploit the continuous range of excitation frequencies, the IM_CH_f $\alpha$ images deliberately resemble those derived by multi-tone signals in order to perform a more reliable comparison. Figure 7 shows the results of the ii) and iii) processing for the sample \#4 after the application of the filtering procedure.

\subsection{Selection of the figure of merit}

Since the various obtained images exhibit different scales, depending on the various image formation algorithms, in order to fair evaluate and compare them, a scale-free figure of merit was defined and introduced. It is based on an intuitive notion of "defect image quality".

Precisely, for each image "IM", a SNR value was defined as:

$$
\operatorname{SNR}(\mathrm{IM})=\frac{\max \left\{\mathrm{IM}_{\Gamma}\right\}-\operatorname{mean}\left\{\mathrm{IM}_{\bar{\Gamma}}\right\}}{\operatorname{std}\left\{\mathrm{IM}_{\bar{\Gamma}}\right\}}=\frac{\mu_{\mathrm{IM}}}{\sigma_{\mathrm{IM}}}
$$

where $\mathrm{IM}_{\Gamma}$ and $\mathrm{IM}_{\bar{\Gamma}}$ are complementary parts of the image containing and not containing the defect respectively.

In can be highlighted that the higher the SNR value, the clearer the effect of the defect presence on the probe response and the higher the possibility to detect it.

A graphic explanation of the proposed figure of merit is reported in Figure 8.
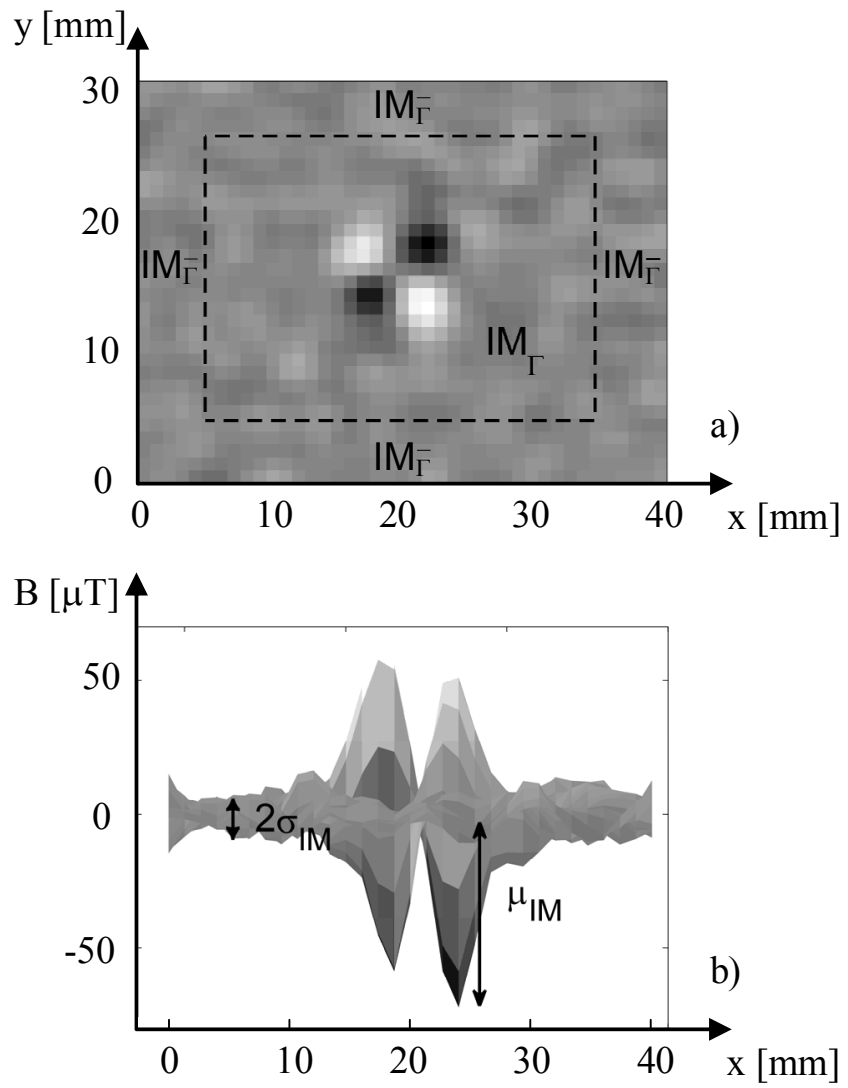

Figure 8. The selected figure of merit. The region of interest (a), and meaning of the $\mu \mathrm{IM}$ and $\sigma \mathrm{IM}$ parameters (b).

\subsection{Results and comments}

Tests were carried out on the five samples described in section III. Table 2 shows the obtained results, in terms of comparison of different excitation strategies and different processing solutions, by using the selected figure of merit (SNR(IM)). Some considerations can be made: a) the value of SNR depends on the values of length, depth and height of the crack; b) the higher the crack height the higher the SNR values whatever the type of processing and defect length. This can be highlighted looking at rows of samples $\# 2$ and \#5; c) the type of chirp processing strongly affects the SNR and it is not possible to identify a processing that is always the best; d) fixing both the crack height and depth, the chirp excitation signal seems to be more promising when short cracks (low values of length) are considered while multi-tone obtains better performance in the case of long cracks (look at rows of samples \#2 and \#5).

Table 2. Results obtained for the selected figure of merit.

\begin{tabular}{|c|c|c|c|c|}
\hline \multirow{2}{*}{ SAMPLE } & \multicolumn{4}{|c|}{ SNR(IM) } \\
\cline { 2 - 5 } & Multi-tone & Chirp with method i) & Chirp with method ii) & Chirp with method iii) \\
\hline$\# 1$ & 10 & 12 & 13 & 12 \\
\hline$\# 2$ & 120 & 104 & 123 & 102 \\
\hline$\# 3$ & 52 & 65 & 57 & 52 \\
\hline$\# 4$ & 7 & 11 & 8 & 64 \\
\hline$\# 5$ & 71 & 63 & 70 & \\
\hline
\end{tabular}




\section{CONCLUSIONS}

The paper presented a preliminary experimental comparison between two of the most adopted complex excitation signals for Non Destructive Eddy Current Testing (ND-ECT). Tests were carried out on a reliable measurement setup and on specimens with reference cracks representing very difficult cases (short and annealed cracks). The introduction of a suitable figure of merit allowed a preliminary quantitative comparison to be carried out. As expected it has been demonstrated that it is not possible to identify an excitation signal that is always the best. In particular, different behaviors between multi-tone and chirp excitation signals are related to the geometrical dimensions of the crack. In addition, as for the chirp signal, also the processing strategy might lead to very different results. Future work will concern the introduction of further figures of merit and analyses able to strictly relate the choice of the best excitation strategy with the crack characteristics. Extending the comparison to other complex signals, the final aim is to realize an ND-ECT instrument with an auto selection capability of the best excitation method able to improve the crack detection and geometrical characterization.

\section{REFERENCES}

[1] D.C. Jiles, "Review of magnetic methods for nondestructive evaluation (Part 2)” NDT Int., vol. 23, pp. 83-92, 1990.

[2] A. Bernieri, G. Betta, L. Ferrigno, M. Laracca, "Crack Depth Estimation by Using a Multi-Frequency ECT Method," IEEE Transactions on Instrumentation and Measurement, Vol. 62, Issue: 3, pp. 544-552, 2013.

[3] Pollakowski, Martin, and Helmut Ermert. "Chirp signal matching and signal power optimization in pulse-echo mode ultrasonic nondestructive testing."Ultrasonics, Ferroelectrics and Frequency Control, IEEE Trans. on 41.5 (1994): 655-659.

[4] K. A. Bartels, J. L. Fisher "Multifrequency eddy current image processing techniques for nondesctructive evaluation " NDE Science and Technology Division IEEE 1995.

[5] P. Burrascano, M. Carpentieri, A. Pirani, M. Ricci "Galois sequences in the non-destructive evaluation of metallic materials”, Meas. Sci. Technol, vol. 17, pp. 2973-2979, 2006.

[6] P. Burrascano, M. Carpentieri, A. Pirani, M. Ricci, F. Tissi, "Time domain deconvolution approach relying on Galois sequences", COMPEL: The International Journal for Computation and Mathematics in Electrical and Electronic Engineering, Vol. 26, Issue 2, pp. 380-388, 2007.
[7] Yin, Wuliang, and A. J. Peyton. "Thickness measurement of non-magnetic plates using multi-frequency eddy current sensors." Ndt \& E International 40.1 (2007): 43-48.

[8] Min, M., et al. "Broadband excitation for short-time impedance spectroscopy." Physiological Measurement 29.6 (2008): S185.

[9] Guang Yang et al., "Pulsed Eddy-Current Based Giant Magnetoresistive System for the Inspection of Aircraft Structures," IEEE Trans. Magn., Vol. 46, N. 3, pp. 910-917, March 2010.

[10] D. Martin et al. , "Multi-modal defect detection of residual oxide scale on a cold stainless steel strip" Machine Vision And Applications 21, pp. 653-666, 2010.

[11] J. Gao, M. Pan, F. Luo, "Defect Identification and Classification of Multi-frequency Eddy Current Test based on Spectrum Method,” in Proc. 2010 IEEE Int. Conf. on Information and Automation, Harbin, China, 2010, pp. 1883-1886.

[12] Guang Yang et al., "Pulsed Eddy-Current Based Giant Magnetoresistive System for the Inspection of Aircraft Structures,” IEEE Trans. Magn., Vol. 46, N. 3, pp. 910917, March 2010.

[13] A. Pirani, M. Ricci, R. Specogna, A. Tamburrino, F. Trevisan,"Multi-frequency identification of defects in conducting media", Inverse Problems, vol. 24, 035011, 2008

[14] A. Bernieri, G. Betta, L. Ferrigno, M. Laracca, "Improving performance of GMR sensors," Sensors Journal, IEEE, vol.13, N.11, pp. 4513-4521, 2013.

[15] G. Betta, P. Burrascano, L. Ferrigno, M. Laracca, M. Ricci, G. Silipigni, "On the Use of Complex Excitation Sequences for Eddy Current Testing," Proceeding of 19th IMEKO TC4 Symposium, Barcelona, Spain, pp. 410-415, July 18-19 2013.

[16] M. Morozov, G.Y. Tian, D. Edgar, "Comparison Of Pec And Sfec Nde Techniques", Nondestructive Testing and Evaluation Vol. 24, Iss. 1-2, 2009

[17] M. R. Schroeder, "Synthesis of Low-Peak-Factor Signals and Binary Sequences With Low Autocorrelation", IEEE Transactions on Information Theory Vol. 16, Issue 1, pp. 85-89, 1970.

[18] [online] NVE Magnetic Sensor Catalog, www.nve.com/sensorcatalog.php

[19] G. Betta, L. Ferrigno, M. Laracca, "GMR-based ECT Instrument for Detection and Characterization of Crack on Planar Specimen: a Hand-held Solution”, IEEE Trans. Instrum. Meas., Vol. 61, No. 2, pp. 505-512, 2012

[20] C.H. Smith, R.W. Schneider, V.A. Pohm, "High-resolution giant magnetoresistance on-chip arrays for magnetic imaging”, Journal of Applied Physics 93, (10), pp. 6864-6866, 2003. 